\title{
Efectos de la retroalimentación auditiva retardada en los patrones de tartamudez
}

\author{
Effects of Delayed Auditory Feedback in Stuttering Patterns \\ Efeitos da retroalimentação auditiva retardada nos patrões de tartamudez \\ Janeth Hernández Jaramillo MSc, PhD (s),' Yelitza Gil Lozada² \\ Recibido: 15 de febrero de 2013 • Aceptado: 7 de diciembre de 2014 \\ Doi: dx.doi.org/10.12804/revsalud12.2.2014.09
}

Para citar este artíiculo: Hernández-Jaramillo J, Gil-Lozada Y. Efectos de la retroalimentación auditiva retardada en los patrones de tartamudez. Rev Cienc Salud. 2014;12(2):243-52. doi: dx.doi.org/10.12804/revsalud12.2.2014.09

\section{Resumen}

El presente estudio se corresponde con un diseño de sujeto único, analiza los patrones de disfluencia en el corpus de habla, en diferentes tareas de lenguaje oral, en las condiciones de uso y no uso de la retroalimentación auditiva retardada (RAR), con el propósito de establecer su efecto en la frecuencia de aparición y tipo de disritmias. El estudio concluye el efecto positivo de la RAR, con un porcentaje de ganancia del $25 \%$ sobre los errores de fluidez, con variación dependiente del tipo de tarea de producción oral. Ello, a su vez, sugiere que el $75 \%$ de las disfluencias, o bien está vinculado con fallos superiores de encodificación o no son susceptibles a resolverse o compensarse mediante la RAR. Los autores discuten las repercusiones de estos hallazgos sobre la intervención terapéutica en tartamudez.

Palabras clave: tartamudez, habla, fluidez, retroalimentación auditiva retardada.

\section{Abstract}

The present study corresponds to a single subject design, analyzes the patterns of stuttering in the speech corpus in various oral language tasks, under the conditions of use or non-use of Delayed Auditory Feedback (DAF), in order to establish the effect of the DAF in the frequency of occurrence and type of dysrhythmia. The study concludes the positive effect of the DAF, with a rate of

\footnotetext{
1 Programa de Fonoaudiología, Escuela de Medicina y Ciencias de la Salud. Universidad del Rosario. Correspondencia: blanca.hernandez@urosario.edu.co

2 Estudiante del Programa de Fonoaudiología, Escuela de Medicina y Ciencias de la Salud. Universidad del Rosario.
} 
return of $25 \%$ on the errors of fluency, with variation depending on the type of oral production task. This in turn suggests that $75 \%$ of the disfluency or linked with top encode failures or not susceptible to resolve or compensated by the DAF. The authors discuss the implications of these findings for therapeutic intervention in stuttering.

Keywords: stuttering, speech fluency, delayed auditory feedback.

\section{Resumo}

O presente estudo se corresponde com uma criação de sujeito único, analisa os patrões de disfluência no corpus de fala, em diferentes tarefas de linguagem oral, nas condições de uso ou não uso da Retroalimentação Auditiva Retardada (RAR), com o propósito de estabelecer o efeito da RAR na frequência de aparição e tipo de disritmias. O estudo conclui o efeito positivo da RAR, com uma porcentagem de ganância dos $25 \%$ sobre os errores de fluidez, com variação dependente do tipo de tarefa de produção oral. Isso a sua vez sugere que o $75 \%$ das disfluências, ou está vinculado com falhas superiores de encodificação, ou não são suscetíveis a se resolver ou compensar mediante a RAR. Os autores discutem as repercussões destas descobertas sobre a intervenção terapêutica em tartamudez.

Palavras-chave: tartamudez, fala, fluidez, retroalimentação auditiva retardada.

\section{Introducción}

La tartamudez es un desorden que afecta directamente la fluidez del habla, en ausencia de compromisos en el pensamiento, dado que el individuo tiene conocimiento exacto de lo que quiere decir (1). Este trastorno se caracteriza por repeticiones o prolongaciones de sonidos, sílabas, palabras y partes de frases, pausas o bloqueos y comportamientos concomitantes (ej. gestos, movimientos compensatorios, tics) que interrumpen la producción de las oraciones. La American Speech-Language-Hearing Association (ASHA) define tartamudez como un trastorno de inicio en la infancia, el cual afecta la fluidez del habla y se caracteriza por interrupciones en la producción de los sonidos (2). Este trastorno puede impactar en diferentes aspectos de la vida (ej. la comunicación, la conducta, la calidad de vida y la auto-aceptación, entre otras) con consecuente restricción en la participación social (3). El Manual diagnóstico y estadístico de los desórdenes mentales IV (DSM-IV por su sigla en inglés) clasifica la tartamudez como un trastorno de inicio en la infancia, la niñez y la adolescencia, y lo subclasifica dentro de los trastornos de la comunicación. El DSM-IV define la tartamudez como una alteración de la fluidez y de la organización temporal normal del habla, con la ocurrencia de repeticiones, prolongaciones, interjecciones y bloqueos (4).

La tartamudez se origina de manera espontánea en el $4 \%$ de los niños, de los cuales el $75 \%$ se recupera durante la infancia, y el $25 \%$ restante sigue presentando episodios de difluencia durante la adultez (5). Es posible que este trastorno disminuya de manera paulatina, a lo largo del tiempo, siendo menos prevalente en la edad adulta, en la medida en que el fonoaudiólogo intervenga oportuna y adecuadamente. Sobre su naturaleza, se ha planteado, además de correlaciones de origen genético, la influencia de factores ambientales 
que precipitan el desencadenamiento de los desórdenes de la fluidez (5).

Existe información sobre su origen genético que indica una mayor incidencia de la tartamudez en familias de quienes presentan este trastorno. Para comprobar esta teoría también se han realizado estudios con gemelos monocigotos y dicigotos, encontrándose una mayor incidencia de la tartamudez en gemelos idénticos, lo cual indica que existe un componente genético (5). Aunque mucho se ha especulado en el pasado sobre su origen psicológico, lo cierto es que las personas con tartamudez presentan dificultades para comunicarse de manera efectiva con los demás, y ello influye negativamente en su desempeño social, ocupacional y emocional. A través de la Clasificación del Funcionamiento, la Discapacidad y la Salud (CIF) es posible identificar los dominios y áreas de la vida que pueden ser afectadas en un individuo con desórdenes de la fluidez. Para el caso específico de la tartamudez, se ha publicado un conjunto de códigos que pueden ser empleados para describir la situación de funcionamiento y restricciones en la participación de quienes presentan este tipo de compromiso en su comunicación; con especial énfasis en las dificultades que experimentan las personas con tartamudez al interactuar con otros en diferentes entornos sociales. Las clasificaciones incluyen actividades tales como: participar en debates, hablar por teléfono, ir de compras, expresar sentimientos, entre otros (3).

La terapia de la tartamudez es un hecho controversial, debido a la escasa proporción de estudios que documentan la eficacia de las intervenciones, los efectos directos sobre la tartamudez y los criterios metodológicos de los tratamientos (6-8). En una revisión sobre la investigación en el tratamiento de la tartamudez, los autores ofrecen una síntesis de los principales hitos que marcaron las últimas cuatro décadas pasadas (6): en la década de los sesenta, la orientación comportamental en el tratamiento de la tartamudez introducía una serie de principios y prácticas para determinar la eficacia de las intervenciones, centrada en la cuantificación de metas terapéuticas alcanzadas, sin mayores repercusiones teóricas. En la década de los setenta, aunque de forma equívoca, por la insuficiencia en los datos, aparecen reportes sobre los porcentajes de éxito de las intervenciones en tartamudez, que incluso hablaban de casi un $90 \%$ de recuperación del total de casos. En los ochenta, otra idea igual de controversial fue publicada, que hacía menciones al carácter 'incurable' en dos de cada cinco adolescentes y adultos con tartamudez. En los noventa, los investigadores y clínicos llaman la atención sobre la necesidad de realizar evaluaciones sistematizadas que permitan levantar evidencia sobre el efecto de diferentes tipos de tratamientos en los diversos comportamientos disfluentes y subgrupos de individuos (ej., niños en edad escolar, adolescentes adultos, monolingües, bilingües). Entrado el 2000, los vacíos en la investigación del tratamiento de la tartamudez se relacionaban con las variables básicas que permitieran establecer puntualmente la eficacia de algún tratamiento (ej., motivación, hábitos del usuario, necesidades específicas, preocupaciones, expectativas) (6).

Entre las opciones de tratamiento, el de tipo farmacológico ha mostrado resultados positivos solo en una reducción del $5 \%$ de los errores de fluidez; fármacos como sertraline y haloperidol registran un porcentajes de reducción entre el $12 \%$ y el $15 \%$ de los episodios de difluencias por un lapso determinado de semanas y en demandas comunicativas específicas (9). Una revisión sistemática de los resultados en el manejo de la tartamudez indica que los beneficios de los programas intensivos son mayores, en particular aquellos que incluyen un habla lenta 
y prolongada, fonación continua y consejería sobre reacciones frente a la tartamudez (10).

Una de las estrategias de intervención que ha venido cobrando protagonismo, con la incorporación de las tecnologías de alta complejidad, es la retroalimentación auditiva retardada (RAR). El uso de la RAR en la intervención de la tartamudez surge del hecho de que la producción del habla está continuamente regulada por el entorno acústico (11). Este efecto es conocido como el efecto de Lombard, el cual consiste en elevar de manera inconsciente el nivel de voz cuando se está en entornos ruidosos. Es a partir de este hallazgo que se ha utilizado del enmascaramiento auditivo como complemento terapéutico para la tartamudez, mediante el uso de sonidos, a determinadas frecuencias e intensidades, hasta lograr un aumento de la fluidez. Existe también evidencia que indica que las respuestas neurales difieren al escuchar la propia voz o la de terceros, mostrándose una mayor activación del lóbulo temporal del hemisferio derecho para el primer caso $(12,13)$.

El papel que desempeña la RAR sobre los mecanismos de producción del habla ha señalado, para algunos casos, reducción de las tasas de disritmias del $31 \%$ (14). El efecto de la RAR consiste en que el hablante oye su propia voz con un retardo mínimo de tiempo, que conduce a una reducción de la velocidad del habla y a la prolongación de las vocales, lo cual provoca una disminución del número de instancias de disfluencia (15). Por esta razón, se ha implementado el uso de RAR como dispositivo tecnológico en el proceso terapéutico de los desórdenes de la fluidez y como una herramienta clínica en el curso de su tratamiento. La utilización del dispositivo de RAR implica la consideración de factores inherentes al usuario tales como el sexo, la edad, la severidad y el origen del trastorno; así como de variables externas relacionadas con el tiempo del retardo en la retroalimentación auditiva, la intensidad, la forma de conducción de la retroalimentación y el tipo de demanda (ej., lectura en voz alta, conversaciones, repetición de frases o palabras, etc.). Finalmente, también se debe tener en cuenta, en la práctica clínica, temas de accesibilidad como el precio, los efectos a largo plazo, la potabilidad y la ergonomía de los dispositivos para RAR (16). Aun así, la evidencia científica sobre su efecto real en tartamudez no es concluyente. Los resultados muestran una alta variabilidad entre individuos y demandas de habla en la disminución o no de los episodios de disfluencia (17).

Un estudio realizado con ocho participantes con tartamudez (18) examinó el efecto del dispositivo RAR sobre la naturalidad del habla y la proporción de sílabas tartamudeadas. Se llevaron a cabo tareas de lectura y monólogos, con y sin la utilización del dispositivo, en forma monoaural, en tres momentos diferentes en un periodo de cuatro meses. Al finalizar el estudio, se registró una reducción en los episodios de disfluencias, no replicable en todos los individuos y con variación según el tipo de tarea lingüística realizada (18). Otro estudio en el cual participaron nueve personas con tartamudez, con el objetivo de investigar el efecto y la percepción de los participantes frente a la exposición repetida y al uso de la RAR durante un periodo de tres meses fuera del entorno clínico, mostró como resultados un aumento de la fluidez durante la utilización de RAR y una mayor seguridad al enfrentar determinadas situaciones comunicativas. Este mismo estudio concluye que existe una reducción inmediata de los episodios de disfluencia mediante el uso de la RAR, pero que son necesarias la orientación y las recomendaciones del fonoaudiólogo especialista durante su utilización, y que la fluidez continúa promoviéndose durante los periodos prolongados de utilización de la RAR, aunque no se presenta evidencia de una relación lineal con el tiempo de exposición (16). 
Un estudio con un solo sujeto, sobre el uso de la RAR, en un hombre de 49 años con tartamudez, luego de una lesión cerebral traumática, concluyó que no existe un patrón de aparición de los errores (repetición de sílabas y palabras, pausas, prolongaciones) en tareas lingüísticas diversas (lectura y habla espontánea) durante el uso o no del dispositivo; y que, por lo tanto, no se aporta a la efectividad del mismo en casos de tartamudez adquirida, de esta manera se resaltan hallazgos que apoyan la diferencia de este tipo de tartamudez con aquella evolutiva o que se da en el desarrollo (19).

La evidencia revisada no permite identificar claramente si existe o no una ganancia real en el porcentaje de fluidez mediante el uso de la RAR, ni para el caso de haberla, cuál es su magnitud; y, finalmente, no es posible decir cuáles tipos de errores en la fluidez del habla se resuelven con RAR y cuáles no.

El presente estudio se corresponde con uno de caso, que compara el perfil de características del habla y el patrón de tartamudez con y sin el uso de RAR. Se analiza el efecto de variables lingüísticas tales como la frecuencia léxica, la frecuencia silábica, la longitud y la estructura de la palabra sobre el tipo de error.

\section{Materiales y métodos}

Las muestras de habla fueron audio-grabadas mediante el uso de una grabadora Sony ${ }^{\circledR}$, computador de escritorio, auriculares y micrófono de alta fidelidad, y el software libre para la utilización de la RAR. Esta última fue provista de forma biaural, con un volumen del $70 \%$ (equivalente a habla conversacional), un retardo de 120 milisegundos y con un tono medio de acuerdo al perfil de voz del participante. Se realizaron todas las calibraciones siguiendo la guía del dispositivo. Fueron levantados los registros audiograbados del habla espontánea, la descripción de una lámina de acción estándar
(Test de Boston lámina de 'robo de galletas'), la repetición de palabras, frases y sílabas, la producción de series verbales automáticas, el canto de un fragmento lírico (a una sola voz y a dos voces), y la lectura en voz alta, silente y subarticulada.

\section{Participantes:}

Las muestras de habla fueron obtenidas de un individuo de sexo masculino, de 16 años de edad, quien cursa tercer año de educación secundaria, con diagnóstico de tartamudez evolutiva moderada, calificada mediante la escala de Riley (20). En su historia se excluyen desórdenes del pensamiento o del aprendizaje. El joven y sus padres aceptaron libremente, mediante asentimiento y consentimiento informado, participar del estudio.

\section{Procedimientos:}

La obtención del corpus de habla fue realizada en un consultorio habilitado, privado, en escenario clínico-hospitalario, confortable, en ausencia de ruido y con suficiente luz. La recolección de la información se hizo conforme a la Guía de Práctica para el Tratamiento de la Tartamudez de la American Speech-LanguageHearing Association (ASHA) (21). Se realizó observación y registro de los comportamientos concomitantes al habla disfluente durante la entrevista. Para la toma de muestras se llevó a cabo una distribución aleatoria de las tareas orales, con y sin el uso del dispositivo de habla retardada, con la finalidad de disminuir el efecto del orden de presentación de las demandas de habla. Luego de obtener las muestras, se realizó una transcripción literal y fonético-fonológica del habla, que fueron analizadas por dos investigadores independientes. Fueron identificados el número y el tipo de error de fluidez en ambas condiciones, con y sin la utilización del dispositivo. 


\section{Resultados}

Del conjunto de muestras fueron obtenidas 961 palabras, el total de los errores de disfluencia encontrados en la muestra fue de 130 $(13,52 \%)$. Durante la condición de no utiliza- ción de RAR se cometieron 81 errores de disfluencia $(62,30 \%)$ y 49 errores $(37,69 \%)$ con el uso del dispositivo. En la tabla 1 se especifican los tipos de errores de disfluencia que se encontraron en la muestra con y sin el uso de la RAR.

Tabla 1. Análisis del tipo de error frente a la ưtilización o no de la RAR

\begin{tabular}{lcccc}
\hline \multirow{2}{*}{\multicolumn{1}{c}{ Tipo de error }} & \multicolumn{2}{c}{ No RAR } & \multicolumn{2}{c}{ Sí RAR } \\
\cline { 2 - 5 } & $\mathrm{N}^{\circ}$ & $\%$ & $\mathrm{~N}^{\circ}$ & $\%$ \\
\hline RF: Repetición de frases & 1 & 0,7 & 4 & 3,0 \\
RS: Repetición de sílabas & 8 & 6,15 & 5 & 3,84 \\
B: Bloqueos & 0 & 0 & 0 & 0 \\
RP: Repetición de palabras & 12 & 9,23 & 8 & 6,15 \\
RFO: Repetición de fonemas & 8 & 6,15 & 4 & 3,0 \\
I: Interjección & 4 & 3,0 & 1 & 0,7 \\
RPP: Repetición de partes de la palabra & 5 & 3,84 & 2 & 1,53 \\
P: Prolongaciones & 19 & 14,61 & 4 & 3,07 \\
AD: Adición de palabra & 1 & 0,7 & 2 & 1,53 \\
CA: Cambio de palabra & 1 & 0,7 & & \\
Ca: Cambio de parte de palabra & 1 & 0,7 & & \\
T: Titubeos o pausas & 21 & 16,15 & 19 & 14,61 \\
Totales & 81 & 61,93 & 49 & 37,43 \\
\hline
\end{tabular}

Con la finalidad de identificar cuántos de es- y de contenido, se realizó un análisis de frecuentos errores correspondían a palabras de función cia de errores por categoría de palabra (tabla 2).

Tabla 2. Tipo de palabra vs utilización o no de RAR

\begin{tabular}{lcccc}
\hline \multirow{2}{*}{ Tipo de palabra } & \multicolumn{2}{c}{ No RAR } & \multicolumn{2}{c}{ Sí RAR } \\
\cline { 2 - 5 } & $\mathrm{N}^{\circ}$ & $\%$ & $\mathrm{~N}^{\circ}$ & $\%$ \\
\hline Palabras de función & 39 & 30 & 25 & 12,23 \\
Palabras de contenido & 43 & 33,07 & 24 & 18,46 \\
\hline
\end{tabular}


Debido a la variabilidad por tipo de demanda lingüística presente en otros estudios, se realizó una comparación entre tareas. La tabla 3 presenta la relación de número de errores cometidos por tipo de tarea, en función de la utilización o no de RAR, con un mayor efecto para la lectura en voz alta.

\section{Tabla 3. Tipo de tarea realizada y número de errores cometidos sin y con RAR}

\begin{tabular}{lcccc}
\hline \multirow{2}{*}{ Tipo de tarea } & \multicolumn{2}{c}{ No RAR } & \multicolumn{2}{c}{ Sí RAR } \\
\cline { 2 - 5 } & $\mathrm{N}^{\circ}$ & $\%$ & $\mathrm{~N}^{\circ}$ & $\%$ \\
\hline Lectura & 51 & 39,23 & 31 & 23,84 \\
Narración & 22 & 16,92 & 9 & 6,92 \\
Repetición & 0 & 0 & 4 & 3,07 \\
Series verbales & 8 & 6,15 & 5 & 3,84 \\
\hline
\end{tabular}

Fue realizado un análisis en función de la frecuencia léxica, para lo cual el total de errores fue procesado mediante el uso de la base de datos BuscaPalabras (22). En la tabla 4 se muestra el efecto por frecuencia de las palabras en el total de errores para ambas condiciones, con una disminución importante en el porcentaje de error sobre la alta frecuencia léxica en la condición de uso de la RAR.

Tabla 4. Frecuencia de las palabras sin y con RAR

\begin{tabular}{rcccc}
\hline \multirow{2}{*}{ Frecuencia } & \multicolumn{2}{c}{ No RAR } & \multicolumn{2}{c}{ Sí RAR } \\
\cline { 2 - 5 } & $\mathrm{N}^{\circ}$ & $\%$ & $\mathrm{~N}^{\circ}$ & $\%$ \\
\hline Alta frecuencia & 20 & 25 & 13 & 26,53 \\
Baja frecuencia & 61 & 75,30 & 36 & 76,46 \\
\hline
\end{tabular}

En la tabla 5 aparece la información obtenida en el análisis de la estructura y longitud de las palabras-error, con un incremento en el número de errores en las formas consonantevocal (Cv) y en palabras de longitud corta.

Tabla 5. Estructura y longitud de la palabra sin y con RAR

\begin{tabular}{lcccc}
\hline \multirow{2}{*}{$\begin{array}{c}\text { Estructura y longitud de } \\
\text { palabra }\end{array}$} & \multicolumn{2}{c}{ No RAR } & \multicolumn{2}{c}{ Sí RAR } \\
\cline { 2 - 5 } & $\mathrm{N}^{\circ}$ & $\%$ & $\mathrm{~N}^{\circ}$ & $\%$ \\
\hline $\mathrm{CV}$ & 13 & 10 & 10 & 7,6 \\
$\mathrm{CVC}$ & 10 & 7,69 & 3 & 2,3 \\
$\mathrm{VC}$ & 11 & 8,4 & 2 & 1,5 \\
CVCVCV & 5 & 3,84 & 3 & 2,3 \\
\hline
\end{tabular}

CV: Consonante vocal. CVC: Consonante vocal consonante. Vc: Vocal Consonante: CVCVCV: Consonante vocal consonante vocal consonante vocal. 


\section{Discusión}

Los resultados arriba presentados muestran que existe una ganancia del $25 \%$ con la utilización de la RAR pero que esta varía según el tipo de tareas lingüísticas realizadas y la estructura silábica y longitud de las palabras. Este margen es cercano a lo reportado en otro estudio (14) que mostró un rango de disminución del porcentaje de disfluencias entre el $35 \%$ y el $44 \%$, dependiendo del tiempo de retardo del estímulo auditivo, pero también se constató una variabilidad considerable entre los individuos.

También se hallaron diferencias en el porcentaje de ganancia según el tipo de tarea lingüística realizada, con un mayor efecto para el habla narrativa, seguido de lectura en voz alta; lo cual coincide con la variabilidad reportada en otras investigaciones en función de la demanda lingüística $(15-17,23,24)$. En el caso de la lectura, los datos obtenidos también pueden apoyar la hipótesis que los errores de disfluencia que persisten son debidos a fallos en el procesamiento superior, relacionados con procesos de codificación y no obedecen a errores exclusivamente motores (25). Sobre esta apuesta, las teorías psicolingüísticas sugieren que la tartamudez comienza durante la planeación del habla, esto es, mucho antes que la producción oral (26-28). De estas teorías, la hipótesis de reparación cubierta (covert repair hypothesis) propone que las disfluencias son resultado de un déficit en la planeación lenguaje-habla; y que, de hecho, un sistema fonológico lento desencadena un mayor número de errores fonológicos y una alta demanda de auto-reparaciones que interrumpen el fluido del habla (27), que no se resolverían con el uso de la RAR.

Los resultados obtenidos aunque evidencian una disminución del número de errores en la fluidez mediante el uso de la RAR, hay cierto tipo de errores tales como las repeticiones de partes de frase y frases completas que aumentan durante la utilización del dispositivo en comparación con la no utilización del mismo. En este mismo sentido, la RAR no parece resolver otro tipo de fallos en la fluidez que obedecerían a otros niveles de codificación o procesamiento. En todo caso, de ser una opción de intervención en tartamudez, la RAR debe ser usada como complemento del proceso terapéutico y no como remplazo del tratamiento.

Un mayor número de estudios deberían ser conducidos con el objetivo de establecer los mecanismo mediante los cuales la RAR resuelve qué tipo de errores de habla o disritmias, y cuáles otros debieran responder mejor a otro de tipo de estrategias terapéuticas en orden de obtener una ganancia más significativa para aquellos con diagnóstico de tartamudez.

\section{Referencias}

1. Van Borsel J, Sierens S, Pereira MM. Using delayed auditory feedback in the treatment of stuttering: evidence to consider. Pro Fono. 2007;19(3):323-32.

2. American Speech Language Hearing Association. Stuttering [internet]. 2012 [citado 2013 abr 4]. Disponible en: http://www.asha.org/public/speech/disorders/stuttering/

3. Yaruss JS, Quesal RW. Stuttering and the international classification of functioning, disability, and health: an update. J Commun Disord. 2004;37(1):35-52.

4. American Psychiatric Association. Manual diagnóstico y estadístico de los desórdenes mentales DSM IV. Barcelona: Masson; 1995.

5. Ambrose NG. Theoretical Perspectives on the Cause of Stuttering. CICSD. 2004;31:80-91. 
6. Prins D, Ingham RJ. Evidence-based treatment and stuttering historical perspective. J Speech Lang Hear Res. 2009;52(1):254-63.

7. Bothe AK, Davidow JH, Bramlett RE, Ingham RJ. Stuttering treatment research 1970-2005: Systematic review incorporating trial quality assessment of behavioral, cognitive, and related approaches. Am J Speech Lang Pathol. 2006;15(4):321-41.

8. Thomas C, Howell P. Assessing efficacy of stuttering treatments. J Fluency Disord. 2001;26(4):311-33.

9. Bothe AK, Davidow JH, Bramlett RE, Franic DM, Ingham RJ. Stuttering treatment research 1970-2005: II. Systematic Review incorporating trial quality assessment of pharmacological approaches. Am J Speech Lang Pathol. 2006;15(4):342-52.

10. Irani F, Gable R, Daniels D, Hughes S. The long term effectiveness of intensive stuttering therapy: a mixed methods study. J Fluency Disord. 2012;37(3):164-78.

11. Corey DM, Cuddapah VA. Delayed auditory feedback effects during reading and conversation tasks: gender differences in fluent adults. J Fluency Disord. 2008;33(4):291-305.

12. Takaso H, Eisner F, Wise RJ, Scott SK. The effect of delayed auditory feedback on activity in the temporal lobe while speaking: a positron emission tomography study. J Speech Lang Hear Res. 2010;53(2):226-36.

13. Toyomura A, Koyama S, Miyamaoto T, Terao A, Omori T, Murohashi H, et al. Neural correlates of auditory feedback control in human. Neuroscience. 2007;146(2):499-503.

14. Armson J, Kiefte M, Mason J, De Croos D. The effect of SpeechEasy on stuttering frequency in laboratory conditions. J Fluency Disord. 2006;31(2):137-52.

15. O'Donnell JJ, Armson J, Kiefte M. The effectiveness of SpeechEasy during situations of daily living. J Fluency Disord. 2008;33(2):99-199.

16. Van Borsel J, Reunes G, Van den Bergh N. Delayed auditory feedback in the treatment of stuttering: clients as consumers. Int J Lang Commun Disord. 2003;38(2):119-29.

17. Van Borsel J, Sierens S, Pereira MM. Using delayed auditory feedback in the treatment of stuttering: evidence to consider. Pro Fono 2007;19(3):323-32.

18. Stuart A, Kalinowski J, Rastatter M, Saltuklaroglu T, Dayalu V. Investigations of the impact of altered auditory feedback in-the-ear devices on the speech of people who stutter: initial fitting and 4-month follow-up. Int J Lang Commun Disord. 2004;39(1):93-113.

19. Van Borsel J, Drummond D, Medeiros M. Delayed auditory feedback and acquired neurogenic stuttering. J Neuroling. 2010;23(5):479-87.

20. Riley G D. Stuttering severity instrument for children and adults (SSI-3) 3. ${ }^{a}$ ed. Austin: PRO-ED; 1994.

21. Asha.org. Association. Guidelines for Practice in Stuttering Treatment [internet]. 1995. [citado 4 abr 2013]. Disponible en: http://www.asha.org/policy/GL1995-00048/

22. Davis CJ, Perea M. BuscaPalabras: A program for deriving orthographic and phonological neighborhood statistics and other psycholinguistic indices in Spanish. Behav Res Methods. 2005;37(4):665-71.

23. Antipova EA, Purdy SC, Blakeley M, Williams S. Effects of altered auditory feedback (AAF) on stuttering frequency during monologue speech production. J Fluency Disord. 2008;33(4):274-90.

24. Lincoln M, Packman A, Onslow M, Jones M. An experimental investigation of the effect of altered auditory feedback on the conversational speech of adults who stutter. J Speech Lang Hear Res. 2010;53(5):1122-31.

25. Hernández J, Álvarez CJ. La tartamudez como un fenómeno pre-articulatorio. Acta Neurol Colomb. 2009;25(1):25-33.

26. Anderson JD, Conture EG. Language abilities of children who stutter: a preliminary study. J Fluency Disord. 2000;25(4):283-304. 
Hernández-Jaramillo J, Gil-Lozada Y

27. Melnick KS, Conture EG, Ohde RN. Phonological priming in picture naming of young children who stutter. J Speech Lang Hear Res. 2003;46(6):1428-43.

28. Postma A, Kolk H. Error monitoring in people who stutter: evidence against auditory feedback defect theories. J Speech Hear Res. 1992;35(5):1024-32. 